\title{
Anti-PVRIG Monoclonal Antibody COM701
}

National Cancer Institute

\section{Source}

National Cancer Institute. Anti-PVRIG Monoclonal Antibody COM701. NCI Thesaurus. Code C156791.

A humanized, hybridoma monoclonal antibody ag ainst the poliovirus receptor-related immunog lobulin (PVRIG; PVR Related Immunog lobulin Domain Containing Protein; CD112R), with potential immune checkpoint inhibitory and antineoplastic activities. Upon administration, anti-PVRIG monoclonal antibody COM701 targets and binds to PVRIG expressed on cytotoxic T-lymphocytes (CT Ls) and natural killer (NK) cells within the tumor microenvironment (TME). This blocks the interaction of PVRIG with its lig and nectin cell adhesion molecule 2 (poliovirus receptor-related 2; PVRL2; CD112), which is overexpressed on a variety of tumor cell types. Inhibiting the activation of PVRIG, abrog ates the PVRIG-induced inhibition of T-lymphocyte and NK cell activation. This activates CT Ls and NK cells, enhances anti-tumor responses and immune-mediated tumor cell killing, and inhibits tumor cell proliferation. PVRIG, a member of the B7/CD28 family and immune checkpoint receptor that, upon activation, negatively regulates the activation of various immune cells. It plays a key role in immunosuppression. 